UDC 547.327

\title{
SYNTHESIS OF SOME FUNCTIONALIZED PYRIDONES DERIVATIVES
}

\author{
F.N. Naghiyev \\ Baku State University \\ AZ-1148 Baku, Z.Khalilov 23, e-mail: farid.orgchemist@ gmail.com
}

Received 15.07.2019

It was established formation of new substituted pyridone derivativesin terms of addition reaction according to Michael with the participation of 2-cyano-3-(4-pyridul) acrylamide and benzoylacetone or ethyl alcohol in ether of acatecetic acid in the presence (MP) in methanol medium. The boiling of ethyl 5-cyano-2hydroxy-2-methyl-6-oxo-4-phenylpiperidine-3-carboxylate in ethanole during 4 hours followed by dehydration and dehydrogenation (by air oxidation) generated 5-cyano-2-methyl -6-oxo-4-phenyl-1,6dihydropyridine-3-carboxylic acid. Under similar conditions there were synthesized appropriate products of substituted pyridone according to Michael' addition reaction through interacting of 4bromobenzoylacetonitrile with 2-cyano-3-(2-fluorophenyl)acrylamide. Structures of all synthesized compounds were proven by NMR spectroscopy.

Keywords: 2-cyano-3-(4-pyridyl)acrylamide, fluorobenzylidenecyanoacetamide, 4-chloroacetoacetate, 4bromobenzoylacetonitrile

DOI: 10.32737/2221-8688-2019-4-600-606

\section{Introduction}

2-Pyridone, tatomer of 2- dients contain valuable pyridone fragments in hydroxypyridine, is one of the major their composition. Also, synthesis methods of heteroaromatic rings in natural products, these compounds are different from in reaction bioactive molecules and pharmaceutical conditions in literature [1-12]. Some articles ingredients. Ciclopirox, milrinone, campto- [13-17] research into antimicrobial activity of thecin, (-)-citisin, fredericamycin, perampanel, pyridone derivatives. bioactive molecules and pharmaceutical ingre-

\section{Results and discussion}

In the presence of methylpiperazine acetoacetate, 2-cyano-3-(pyridine-4-yl) and methanol medium according to Michael acrylamide at a room temperature was addition reaction of benzoilacetone or ethyl hydroxysubsituted pyridone derivatives.

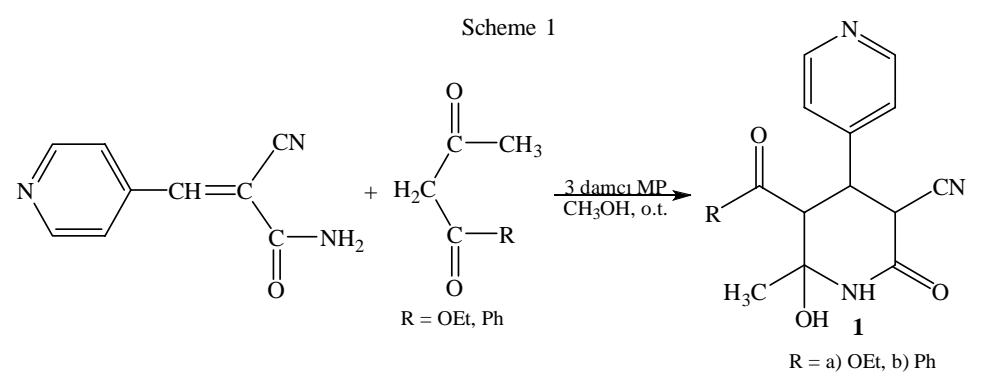

Scheme 1. Reaction of 2-cyano-3-(4-pyridyl)acrylamide with benzoylacetone and ethyl acetoacetate.

In our view, in the initial step of reaction the nucleophilic attack methylpiperazine to methylene-active compound results in the formation of corresponding anion (nucleophilic particle), which, in turn, drew to the $\mathrm{CH}$-electrophilic center of the activated double bound to form intermediate $\mathbf{A}$ (according to Michael adduct). In the final 
step, in the intermediate $\mathbf{A}$, the amide nitrogen desired ring closure product - pyridione attacked the carbonyl group to generate a (Scheme 2).

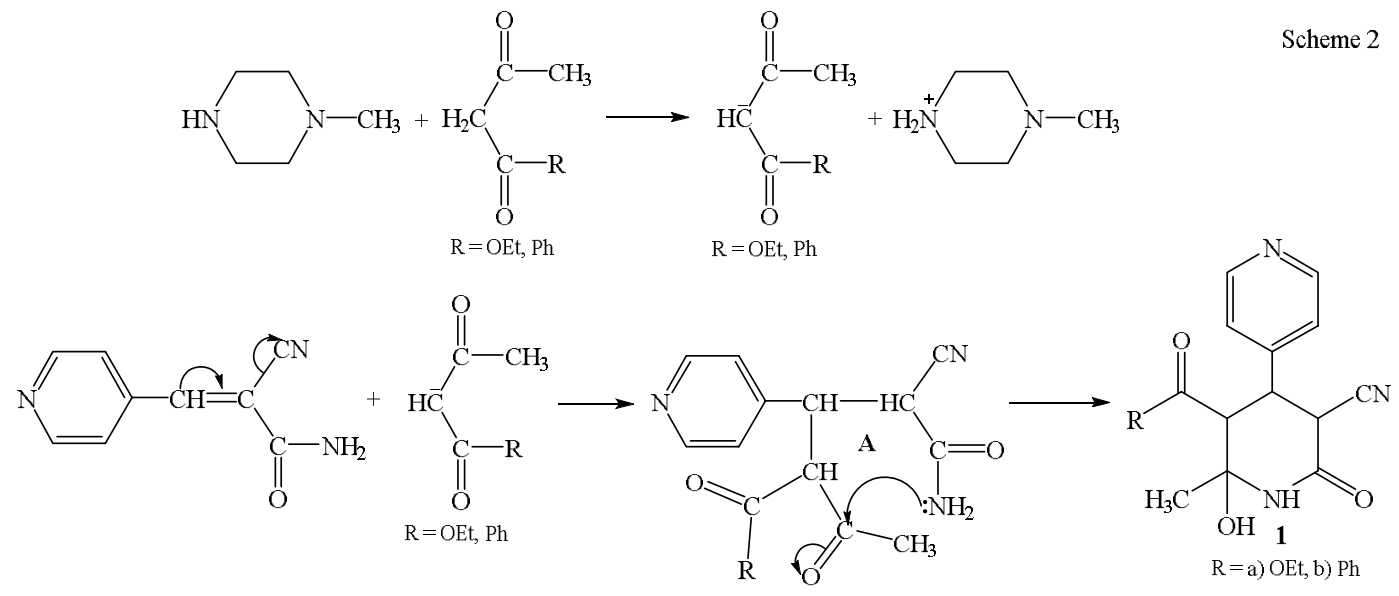

Boiling of ethyl 5-cyano-2-hydroxy-2-methyl- oxidation) to provide 5-cyano-2-methyl -66-oxo-4-phenylpiperidine-3-carboxylate in oxo-4-phenyl-1,6-dihydropyridine-3ethanole for 4 hours was followed by carboxylic acid. dehydration and dehydrogenation (by air<smiles>CCOC(=O)C1C(c2ccncc2)C(C#N)C(=O)NC1(C)O</smiles><smiles>Cc1[nH]c(=O)c(C(=O)O[Ga]2C#CC2)c(-c2ccncc2)c1C#N</smiles>

Scheme 2. Synthesis of ethyl 5-cyano-2-methyl-6-oxo-4-phenyl-1,6-dihydropyridine-3carboxylat

Scheme 4<smiles>N#C/C(=C\c1ccc([N+](=O)[O-])cc1)C(N)=O</smiles><smiles>O=C(O)CC(=O)Cl</smiles><smiles>CCCC(=O)C1C(c2ccc([N+](=O)[O-])cc2)C(C#N)C(=O)NC1(C)O</smiles>

Scheme 3. Reaction of 2-cyano-3-(4-nitrophenyl)acrylamide with ethyl 4-chloroacetoacetate.

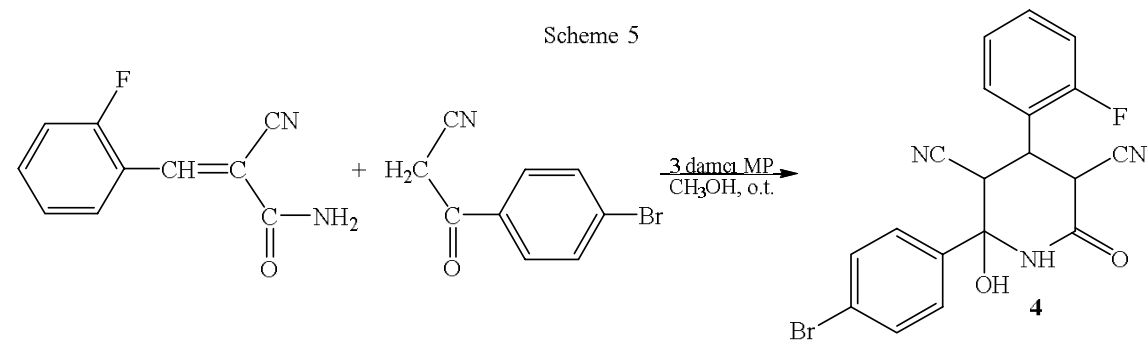

Scheme 4. Reaction of 2-cyano-3-(2-fluorophenyl)acrylamide with 4-bromobenzoylacetonitrile. 
Under the same reaction condition, the Michael addition of 4-bromobenzoylacetonitrile to 2-cyano-3-(2-fluorophenyl)

acrylamide led to the formation of appropriate hydroxy substituted pyridone derivatives.

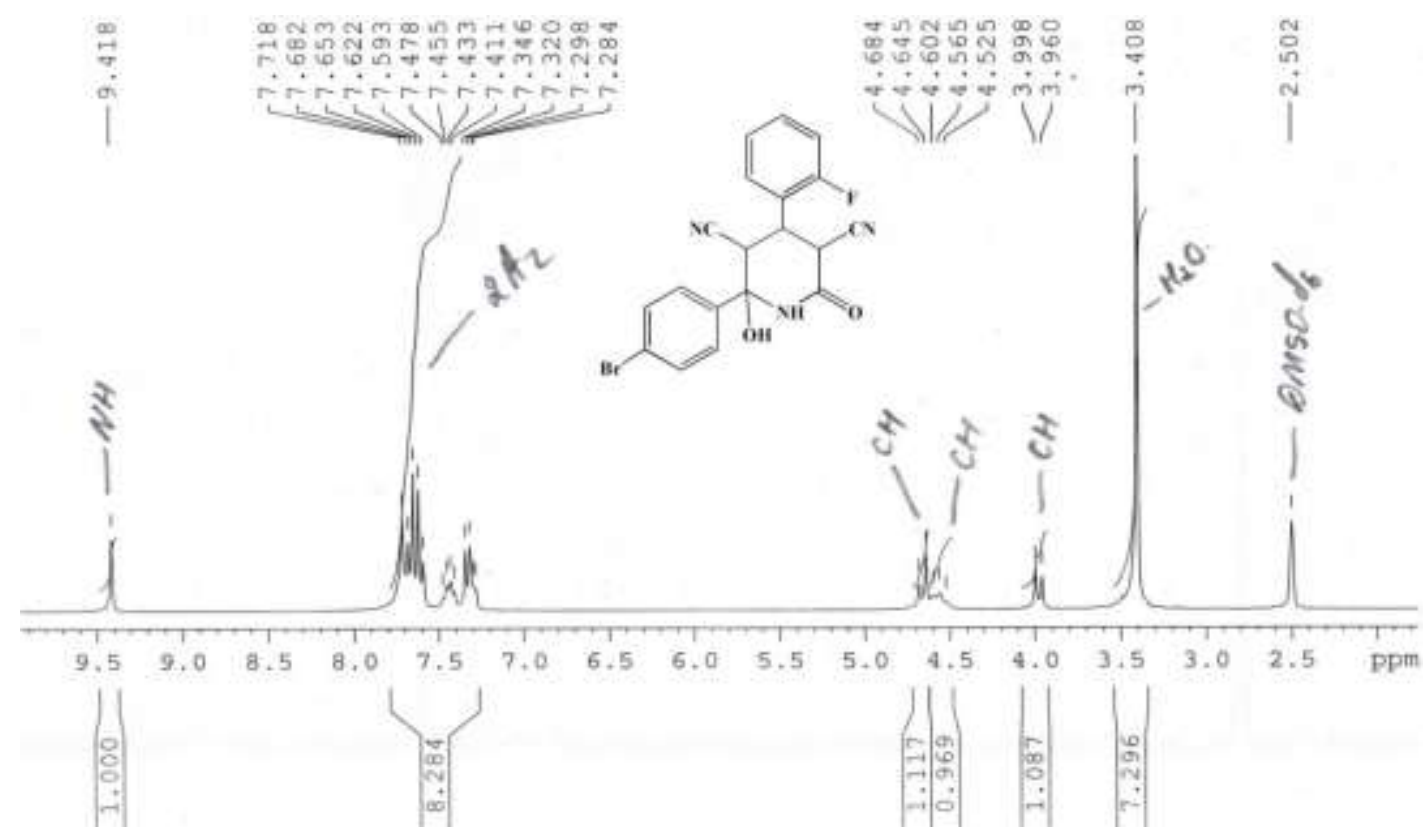

Fig. 1. ${ }^{1} \mathrm{H}$ NMR spectrum of 2-(4-bromophenyl)-4-(2-fluorophenyl)-2-hydroxy-6-oxopiperidine3,5-dicarbonitrile (4)

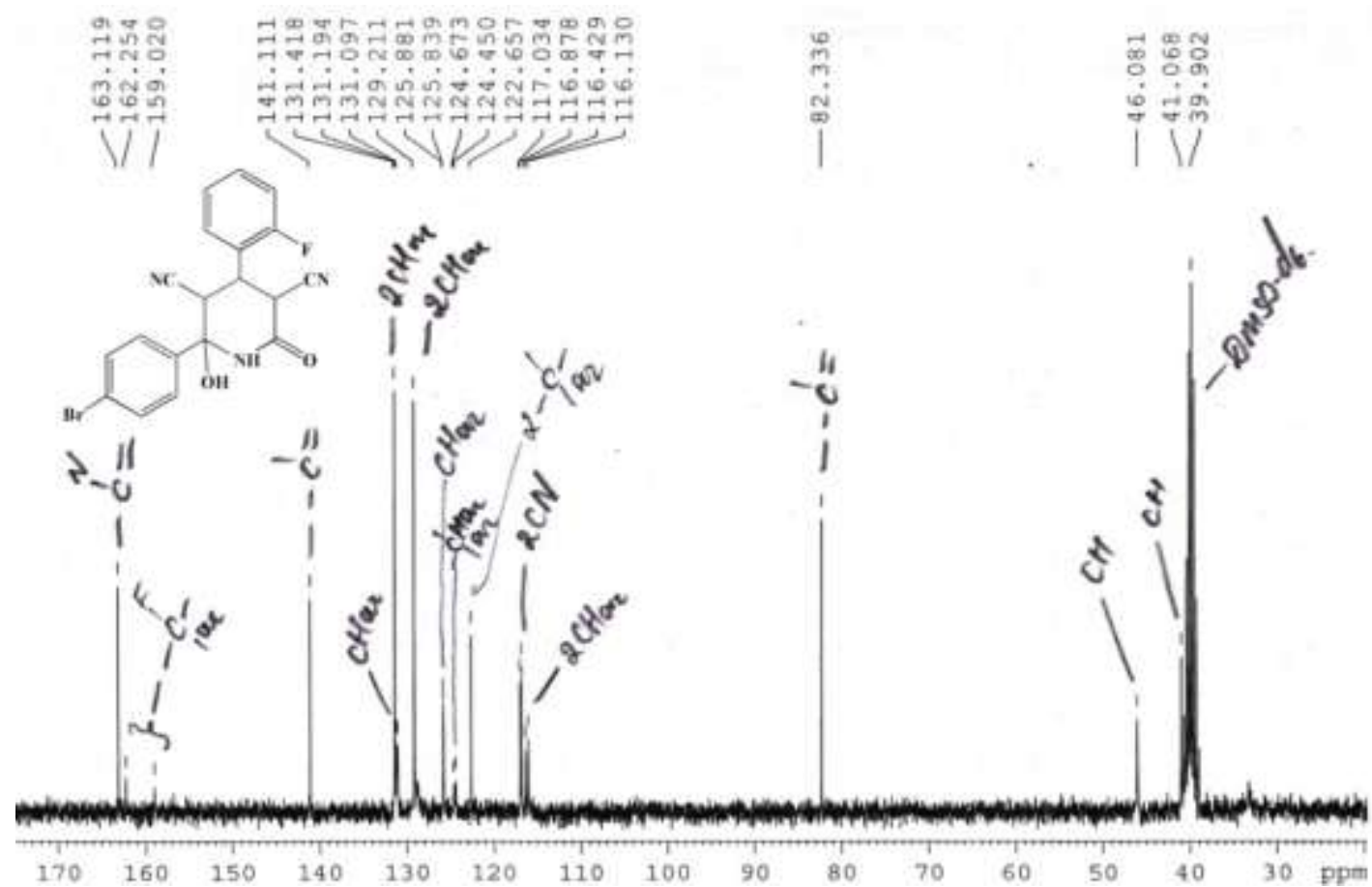

Fig. 2. ${ }^{13} \mathrm{C}$ NMR spectrum of 2-(4-bromophenyl)-4-(2-fluorophenyl)-2-hydroxy-6-oxopiperidine3,5-dicarbonitrile (4) 


\section{Experimental part. General remarks}

All commercially available chemicals were obtained from Merck and Fluka (Sigma Aldrich) companies and used without further purification. Melting points were measured by Stuart SMP30 apparatus without correction. ${ }^{1} \mathrm{H}$, ${ }^{13}$ C NMR spectra (Fig.1) were recorded on BrukerAvance $300-\mathrm{MHz}$ spectrometer at 300 and $75 \mathrm{MHz}$, respectively. Thin-layer chromatography (TLC) on commercial aluminumbacked plates of silica gel (60 F254) was used to monitor the reaction course.

\section{Experimental procedures:}

Ethyl 5-cyano-2-hydroxy-2-methyl-6-oxo-4(pyridin-4-yl)piperidine-3-carboxylate (1a): 2-Cyano-3-(4-pyridyl)acrylamide $(5.1 \mathrm{mmol})$ and ethyl acetoacetate $(5.2 \mathrm{mmol})$ was stirrered in $35 \mathrm{ml}$ of methyl alcohol. Then 3-4 drops of 1-methylpiperazine were added to reaction mixture and stirrered for 5 minutes. Then reaction mixture was held out at a room temperature for $48 \mathrm{~h}$. Reaction course was monitored by TLC (EtOAc/n-hexane, 3:1). Crystals were precipitated after evaporation of solvent, filtered, recrystallized from ethanolwater mixture and obtained in pure form (yield $1.27 \mathrm{~g}, 82.47 \%) . \mathrm{T}_{\mathrm{mp} .}=173^{\circ} \mathrm{C}$.

${ }^{1} \mathrm{H}$ NMR $\left(300 \mathrm{MHz}, \mathrm{DMSO}-d_{6}\right): 0.84(\mathrm{t}$, $\left.3 \mathrm{H}, \mathrm{CH}_{3},{ }^{3} J_{\mathrm{H}-\mathrm{H}}=7\right) ; 1.47\left(\mathrm{~s}, 3 \mathrm{H}, \mathrm{CH}_{3}\right) ; 3.44$ $\left(\mathrm{d}, 1 \mathrm{H}, \mathrm{CH},{ }^{3} J_{\mathrm{H}-\mathrm{H}}=12.4\right) ; 3.82\left(\mathrm{k}, 2 \mathrm{H}, \mathrm{CH}_{2} \mathrm{O}\right.$, $\left.{ }^{3} J_{\mathrm{H}-\mathrm{H}}=6.9\right) ; 3.92\left(\mathrm{t}, 1 \mathrm{H}, \underline{\mathrm{CH}}-\mathrm{Ar},{ }^{3} J_{\mathrm{H}-\mathrm{H}}=12.3\right)$; $4.31\left(\mathrm{~d}, 1 \mathrm{H}, \mathrm{CH},{ }^{3} J_{\mathrm{H}-\mathrm{H}}=12.6\right) ; 6.27(\mathrm{~s}, 1 \mathrm{H}$, $\mathrm{OH}) ; 7.39\left(\mathrm{~d}, 2 \mathrm{H}, 2 \mathrm{CH}_{\text {pyrid., }}{ }^{3} \mathrm{~J}_{\mathrm{H}-\mathrm{H}}=5.1\right) ; 8.55$ $\left(\mathrm{d}, 2 \mathrm{H}, 2 \mathrm{CH}_{\text {pyrid. }},{ }^{3} \mathrm{~J}_{\mathrm{H}-\mathrm{H}}=5.1\right) ; 8.97(\mathrm{~s}, 1 \mathrm{H}$, $\mathrm{NH}) .{ }^{13} \mathrm{C}$ NMR $\left(75 \mathrm{MHz}, \mathrm{DMSO}-d_{6}\right): 14.02$ $\left(\mathrm{CH}_{3} \mathrm{CH}_{2}\right), 28.34 \quad\left(\mathrm{CH}_{3}\right), 40.41 \quad(\underline{\mathrm{CH}}-\mathrm{CN})$, $41.44 \quad(\underline{\mathrm{CH}}-\mathrm{Ar}), \quad 54.24 \quad\left(\underline{\mathrm{CH}}-\mathrm{CO}_{2}\right), \quad 60.64$ $\left(\underline{\mathrm{CH}}_{2} \mathrm{O}\right), 80.72\left(\mathrm{O}-\underline{\mathrm{C}}_{\text {dördlui }}\right), 117.29(\mathrm{CN}), 123.85$ $\left(2 \mathrm{CH}_{\text {pyrid. }}\right), 148.63\left(\mathrm{C}_{\text {pyrid. }}\right), 150.33\left(2 \mathrm{CH}_{\text {pyrid. }}\right)$, $162.56(\mathrm{~N}-\underline{\mathrm{C}}=\mathrm{O}), 168.58(\mathrm{O}-\underline{\mathrm{C}}=\mathrm{O})$.

Found, \%: $59.35 \mathrm{C} ; 5.67 \mathrm{H} ; 13.81 \mathrm{~N}$. $\mathrm{C}_{15} \mathrm{H}_{17} \mathrm{~N}_{3} \mathrm{O}_{4}$. Calculated, \%: $59.40 \mathrm{C} ; 5.61 \mathrm{H}$; $13.86 \mathrm{~N}$.

\section{5-Benzoyl-6-hydroxy-6-methyl-2-oxo-4-} (pyridin-4-yl)piperidine-3-carbonitrile (1b): 2-Cyano-3-(4-pyridyl)acrylamide $(5.1 \mathrm{mmol})$ and benzoylacetone $(5.2 \mathrm{mmol})$ was stirrered in $35 \mathrm{ml}$ of methyl alcohol. After 3-4 drops of 1methylpiperazine added to reaction mixture and stirrered for 5 minutes. Then reaction mixture was held out at a room temperature for $48 \mathrm{~h}$. Reaction course was monitored by TLC (EtOAc/n-hexane, 3:1). Crystals were precipitated after evaporation of solvent, filtered, recrystallized from ethanol-water mixture and obtained in pure form (yield $1.32 \mathrm{~g}$, $77.65 \%) . \mathrm{T}_{\mathrm{mp}} .=192^{\circ} \mathrm{C}$.

${ }^{1} \mathrm{H}$ NMR (300 MHz, DMSO- $\left.d_{6}\right): 1.35(\mathrm{~s}$, $\left.3 \mathrm{H}, \mathrm{CH}_{3}\right) ; 3.86\left(\mathrm{t}, 1 \mathrm{H}, \underline{\mathrm{CH}}-\mathrm{Ar},{ }^{3} J_{\mathrm{H}-\mathrm{H}}=11.4\right.$ ); $4.60\left(\mathrm{~d}, 1 \mathrm{H}, \mathrm{CH},{ }^{3} J_{\mathrm{H}-\mathrm{H}}=12.3\right) ; 4.68(\mathrm{~d}, 1 \mathrm{H}, \mathrm{CH}$, $\left.{ }^{3} J_{\mathrm{H}-\mathrm{H}}=11.1\right) ; 6.46(\mathrm{~s}, 1 \mathrm{H}, \mathrm{OH}) ; 7.44-8.44(\mathrm{~m}$, $\left.9 \mathrm{H}, 5 \mathrm{Ar}-\mathrm{H}+4 \mathrm{CH}_{\text {pyrid. }}\right) ; 8.84(\mathrm{~s}, 1 \mathrm{H}, \mathrm{NH}) .{ }^{13} \mathrm{C}$ NMR (75 MHz, DMSO- $\left.d_{6}\right)$ : $27.01 \quad\left(\mathrm{CH}_{3}\right)$, $40.78(\underline{\mathrm{CH}}-\mathrm{CN}), \quad 42.98 \quad(\underline{\mathrm{CH}}-\mathrm{Ar}), \quad 55.15$ $(\underline{\mathrm{CH}}-\mathrm{C}=\mathrm{O}), 82.86\left(\mathrm{O}-\underline{\mathrm{C}}_{\text {dördlü }}\right), 117.26(\mathrm{CN})$, $123.83\left(2 \mathrm{CH}_{\text {arom. }}\right), 129.04\left(2 \mathrm{CH}_{\text {pirid. }}\right), 129.14$ (2 $\left.\mathrm{CH}_{\text {arom. }}\right), 134.09$ ( $\left.\mathrm{CH}_{\text {arom. }}\right), 138.17\left(\mathrm{C}_{\mathrm{ar}}\right)$, $148.52\left(\mathrm{C}_{\text {pyrid. }}\right), 150.25$ (2 $\left.\mathrm{CH}_{\text {pyrid. }}\right), 162.43$ $(\mathrm{N}-\mathrm{C}=\mathrm{O}), 199.23(\mathrm{C}=\mathrm{O})$.

Found, \%: $68.00 \mathrm{C} ; 5.02 \mathrm{H} ; 12.60 \mathrm{~N}$. $\mathrm{C}_{19} \mathrm{H}_{17} \mathrm{~N}_{3} \mathrm{O}_{3}$. Calculated, \%: $68.06 \mathrm{C} ; 5.07 \mathrm{H}$; $12.54 \mathrm{~N}$.

Ethyl 5-cyano-2-methyl-6-oxo-1,6dihydro-[4,4'-bipyridine]-3-carboxylate (2): Ethyl 5-cyano-2-hydroxy-2-methyl-6-oxo-4(pyridin-4-yl)piperidine-3-carboxylate mmol) was dissolved in $35 \mathrm{ml}$ of ethyl alcohol and $5 \mathrm{~h}$ refluxed. Then the resulting reaction mixture was placed in a glass. Crystals were precipitated after evaporation of solvent, filtered, recrystallized from ethanol-water mixture and obtained in pure form (yield $1.07 \mathrm{~g}$, $74.30 \%) . \mathrm{T}_{\mathrm{mp}}=147^{\circ} \mathrm{C}$.

${ }^{1} \mathrm{H}$ NMR $\left(300 \mathrm{MHz}\right.$, DMSO- $\left.d_{6}\right): 0.70(\mathrm{t}, 3 \mathrm{H}$, $\left.\mathrm{CH}_{3},{ }^{3} J_{\mathrm{H}-\mathrm{H}}=7.2\right) ; 2.45\left(\mathrm{~s}, 3 \mathrm{H}, \mathrm{CH}_{3}\right) ; 3.83(\mathrm{k}$, $\left.2 \mathrm{H}, \underline{\mathrm{CH}_{2}} \mathrm{O},{ }^{3} J_{\mathrm{H}-\mathrm{H}}=7\right) ; 7.36\left(\mathrm{~d}, 2 \mathrm{H}, 2 \mathrm{CH}_{\text {pyrid. }}\right.$, $\left.{ }^{3} J_{\mathrm{H}-\mathrm{H}}=5.1\right) ; 8.71\left(\mathrm{~d}, 2 \mathrm{H}, 2 \mathrm{CH}_{\text {pyrid. }},{ }^{3} J_{\mathrm{H}-\mathrm{H}}=\right.$ 5.1); 12.82 (s, 1H, NH). ${ }^{13} \mathrm{C}$ NMR (75 MHz, DMSO- $\left.d_{6}\right): \quad 13.37 \quad\left(\underline{\mathrm{CH}}_{3} \mathrm{CH}_{2}\right), \quad 19.13$ $\left(\underline{\mathrm{CH}}_{3}-\mathrm{C}=\right), 61.43\left(\underline{\mathrm{CH}}_{2} \mathrm{O}\right), 101.07$ (= $\left.\underline{\mathrm{C}}_{\text {dördlui }}\right)$, $111.09 \quad\left(=\underline{\mathrm{C}}_{\text {dördliu }}\right), \quad 115.42 \quad(\mathrm{CN}), \quad 122.35$ (2 $\left.\mathrm{CH}_{\text {pyrid. }}\right), 144.51$ ( $\left.\mathrm{C}_{\text {pyrid. }}\right), 150.25\left(2 \mathrm{CH}_{\text {pyrid. }}\right)$, $155.45 \quad\left(=\underline{\mathrm{C}}_{\text {dördlü }}\right), \quad 157.53 \quad\left(=\underline{\mathrm{C}}_{\text {dördlü }}\right), \quad 160.00$ $(\mathrm{N}-\underline{\mathrm{C}}=\mathrm{O}), 164.64(\mathrm{O}-\mathrm{C}=\mathrm{O})$.

Found, \%: $63.55 \mathrm{C} ; 4.65 \mathrm{H} ; 14.89 \mathrm{~N}$. $\mathrm{C}_{15} \mathrm{H}_{13} \mathrm{~N}_{3} \mathrm{O}_{3}$. Calculated, \%: $63.60 \mathrm{C} ; 4.59 \mathrm{H}$; $14.84 \mathrm{~N}$.

Ethyl 2-(chloromethyl)-5-cyano-2-hydroxy4-(4-nitrophenyl)-6-oxopiperidine-3-carbo- 
xylate (3): 2-Cyano-3-(4nitrophenyl)acrylamide $(5.1 \mathrm{mmol})$ and ethyl 4chloroacetoacetate $(5.2 \mathrm{mmol})$ stirrered in $35 \mathrm{ml}$ of methyl alcohol. After 3-4 drops of 1methylpiperazine added to reaction mixture and stirrered for 5 minutes. Then reaction mixture hold out at room temperature for $48 \mathrm{~h}$. Reaction course was monitored by TLC (EtOAc/n-hexane, 3:1). Crystals were precipitated after evaporation of solvent, filtered, recrystallized from ethanol-water mixture and obtained in pure form (yield $1.57 \mathrm{~g}$, $80.51 \%) . \mathrm{T}_{\mathrm{mp} .}=209^{\circ} \mathrm{C}$.

${ }^{1} \mathrm{H}$ NMR (300 MHz, DMSO- $\left.d_{6}\right): 0.86$ $\left(\mathrm{t}, 3 \mathrm{H}, \mathrm{CH}_{3},{ }^{3} J_{\mathrm{H}-\mathrm{H}}=6.9\right) ; 3.68\left(\mathrm{~d}, 1 \mathrm{H}, \mathrm{CH},{ }^{3} J_{\mathrm{H}-}\right.$ $\mathrm{H}=12.3) ; 3.69\left(\mathrm{~s}, 3 \mathrm{H}, \mathrm{CH}_{2} \mathrm{Cl}\right) ; 3.85(\mathrm{k}, 2 \mathrm{H}$, $\left.\mathrm{CH}_{2} \mathrm{O},{ }^{3} J_{\mathrm{H}-\mathrm{H}}=7\right) ; 4.10\left(\mathrm{t}, 1 \mathrm{H}, \underline{\mathrm{CH}}-\mathrm{Ar},{ }^{3} J_{\mathrm{H}-\mathrm{H}}=\right.$ $12.3) ; 4.63\left(\mathrm{~d}, 1 \mathrm{H}, \mathrm{CH},{ }^{3} J_{\mathrm{H}-\mathrm{H}}=12\right) ; 7.12(\mathrm{~s}$, $1 \mathrm{H}, \mathrm{OH}) ; 7.70\left(\mathrm{~d}, 2 \mathrm{H}, 2 \mathrm{CH}_{\text {arom. }}{ }^{3} J_{\mathrm{H}-\mathrm{H}}=8.4\right)$; $8.24\left(\mathrm{~d}, 2 \mathrm{H}, 2 \mathrm{CH}_{\text {arom. }},{ }^{3} J_{\mathrm{H}-\mathrm{H}}=8.4\right) ; 9.10(\mathrm{~s}, 1 \mathrm{H}$, $\mathrm{NH}) .{ }^{13} \mathrm{C}$ NMR $\left(75 \mathrm{MHz}, \mathrm{DMSO}-d_{6}\right): 13.97$ $\left(\mathrm{CH}_{3} \mathrm{CH}_{2}\right), 40.13(\underline{\mathrm{CH}}-\mathrm{CN}), 41.31(\underline{\mathrm{CH}}-\mathrm{Ar})$, $48.16 \quad\left(\underline{\mathrm{CH}}_{2} \mathrm{Cl}\right), \quad 49.80 \quad(\underline{\mathrm{CH}}-\mathrm{COO}), \quad 61.06$ $\left(\underline{\mathrm{CH}}_{2} \mathrm{O}\right), 83.03\left(\mathrm{O}-\underline{\mathrm{C}}_{\text {dördlü }}\right), 117.01(\mathrm{CN}), 124.13$ $\left(3 \mathrm{CH}_{\text {arom. }}\right), 130.21 \quad\left(\mathrm{CH}_{\text {arom. }}\right), \quad 147.10 \quad\left(\mathrm{C}_{\text {ar. }}\right)$, $147.47 \quad\left(\mathrm{C}_{\mathrm{ar}}\right), \quad 163.30 \quad(\mathrm{~N}-\mathrm{C}=\mathrm{O}), \quad 167.71$ $(\mathrm{O}-\underline{\mathrm{C}}=\mathrm{O})$.

Found, \%: $50.39 \mathrm{C} ; 4.14 \mathrm{H} ; 11.07 \mathrm{~N}$. $\mathrm{C}_{16} \mathrm{H}_{16} \mathrm{~N}_{3} \mathrm{O}_{6} \mathrm{Cl}$. Calculated, \%: $50.33 \mathrm{C} ; 4.19 \mathrm{H}$; $11.02 \mathrm{~N}$.
2-(4-Bromophenyl)-4-(2-fluorophenyl)-2hydroxy-6-oxopiperidine-3,5-dicarbonitrile

(4): 2-Cyano-3-(2-fluorophenyl)prop-2enamide $(5.1 \mathrm{mmol})$ and 4-bromobenzoylacetonitrile $(5.2 \mathrm{mmol})$ stirrered in $35 \mathrm{ml}$ of methyl alcohol. After 3-4 drops of 1-methylpiperazine were added to reaction mixture and stirrered for 5 minutes. Then reaction mixture were held out at a room temperature for $48 \mathrm{~h}$. Reaction course was monitored by TLC (EtOAc/n-hexane, 3:1). Crystals were precipitated after evaporation of solvent, filtered, recrystallized from ethanol-water mixture and obtained in pure form (yield $1.77 \mathrm{~g}, 84.28 \%$ ). $\mathrm{T}_{\mathrm{mp}}=121^{\circ} \mathrm{C}$.

${ }^{1} \mathrm{H}$ NMR $\left(300 \mathrm{MHz}, \mathrm{DMSO}-d_{6}\right): 3.98(\mathrm{~d}$, $\left.1 \mathrm{H}, \mathrm{CH},{ }^{3} J_{\mathrm{H}-\mathrm{H}}=11.4\right) ; 4.56\left(\mathrm{t}, 1 \mathrm{H}, \mathrm{CH},{ }^{3} J_{\mathrm{H}-\mathrm{H}}=\right.$ $11.5) ; 4.66\left(\mathrm{~d}, 1 \mathrm{H}, \mathrm{CH},{ }^{3} J_{\mathrm{H}-\mathrm{H}}=11.7\right) ; 7.28$ $7.72(\mathrm{~m}, 8 \mathrm{H}, 8 \mathrm{Ar}-\mathrm{H}+\mathrm{OH}) ; 9.42(\mathrm{~s}, 1 \mathrm{H}, \mathrm{NH})$. ${ }^{13} \mathrm{C} \quad \mathrm{NMR} \quad\left(75 \mathrm{MHz}, \quad\right.$ DMSO- $\left.d_{6}\right): 39.90$ $(\underline{\mathrm{CH}}-\mathrm{CN}), 41.07(\underline{\mathrm{CH}}-\mathrm{Ar}), 46.08(\underline{\mathrm{CH}}-\mathrm{CN})$, $82.34\left(\mathrm{O}-\mathrm{C}_{\text {dördlü }}\right), 116.13\left(\mathrm{CH}_{\text {arom }}\right), 116.43$ $\left(\mathrm{CH}_{\text {arom }}\right), 116.88(\mathrm{CN}), 117.03(\mathrm{CN}), 122.66$ $\left(\mathrm{Br}-\mathrm{C}_{\mathrm{ar}}\right), \quad 124.45-124.67 \quad\left(\mathrm{C}_{\mathrm{ar} .}\right), \quad 125.88$ $\left(\mathrm{CH}_{\text {arom }}\right), \quad 129.21 \quad\left(2 \mathrm{CH}_{\text {arom }}\right), \quad 131.10-131.19$ $\left(\mathrm{CH}_{\text {arom }}\right), 131.42 \quad\left(2 \mathrm{CH}_{\text {arom }}\right), 141.11 \quad\left(\mathrm{C}_{\text {ar. }}\right)$, 159.02-162.25 (F- $\left.\underline{\mathrm{C}}_{\mathrm{ar}}\right), 163.12(\mathrm{~N}-\mathrm{C}=\mathrm{O})$.

Found, \%: $55.01 \mathrm{C} ; 3.20 \mathrm{H} ; 10.09 \mathrm{~N}$. $\mathrm{C}_{19} \mathrm{H}_{13} \mathrm{~N}_{3} \mathrm{FBrO}_{2}$. Calculated, \%: $55.07 \mathrm{C} ; 3.14$ $\mathrm{H} ; 10.14 \mathrm{~N}$.

\section{References}

1. Maria A.Vodolazhenko, Nikolay Yu.Gorobets. Synthesis of highly substituted 2-pyridones. Chemistry of Heterocyclic Compounds. 2016, vol. 52, iss.11, pp. 894-896.

2. Zhi-Qiang Liu, Bo-Kai Liu, Qi Wu, Xian-Fu Lin. Diastereoselective enzymatic synthesis of highly substituted 3,4-dihydropyridin-2ones via domino Knoevenagel condensation-Michael additionintramolecular cyclization. Tetrahedron. 2011, vol. 67, iss.50, pp. 9736-9740.

3. Zhiqiang Liu, Lu Tan, Qi Wu, Xianfu Lin. Imidazole-catalyzed Three-component Cascade Reaction for the Facile Synthesis of Highly Substituted 3,4-Dihydropyridin2-one Derivatives. Chin. J. Chem. 2012, vol. 30 , pp. $2343-2348$.
4. Naghiyev F.N. The investigation of michael addition of some ylidenecyanoacetamides with acetoacetanilide and methyl acetopyruvate. Azerbaijan Chemical Journal. 2019, no. 2, pp. 35-39.

5. Maharramov A.M., Asgarova A.R., Musayeva S.A., Rahimova A.G., Akhundova M.A., Mamedov I.G. Synthesis of substituted pyridine derivatives by the three component reaction based on ylidenecyanoacetamides. Chemical Problems. 2019, no.1(17), pp. 8792.

6. Erica L. Lanni, Michael A. Bosscher, Bartel D. Ooms, Christina A. Shandro, Bruce A. Ellsworth, Carolyn E. Anderson. Synthesis of Substituted N-Benzyl Pyridones via an O- to N-Alkyl Migration. 
J. Org. Chem., 2008, vol. 73, no. 16, pp. 6425-6428.

7. Archana B. Pandit, Mahesh M. Savant, Kartik D. Ladva. An Efficient One-Pot Synthesis of Highly Substituted Pyridone Derivatives and Their Antimicrobial and Antifungal Activity. Journal of Heterocyclic Chemistry. 2018, vol. 55, iss. 4, pp. 983-987.

8. Färber M., Osiander H., Severin T. Synthesis of N-substituted 3-hydroxy-2-methyl-4-pyridones and -pyridonimines. Journal of Heterocyclic Chemistry. 1994, vol. 31, iss. 4, pp. 947956.

9. Liquan Tan, Peng Zhou, Cui Chen and Weibing Liu. An efficient method for the construction of polysubstituted 4-pyridones via self-condensation of $\beta$-keto amides mediated by $\mathrm{P}_{2} \mathrm{O}_{5}$ and catalyzed by zinc bromide. Beilstein J. Org. Chem. 2013, no. 9, pp. 2681-2687.

10. Rajib Sarkar, Chhanda Mukhopadhyay. 1Proline catalyzed expeditious multicomponent protocol for the synthesis of fused $\mathrm{N}$-substituted-2-pyridone derivatives in aqueous medium. Tetrahedron Letters. 2014, vol. 55, iss.16, pp. 26182624.

11. IssaYavari, Mohammad J.Bayat. An efficient organocatalytic method for tandem synthesis of functionalized 2pyridones. Tetrahedron Letters. 2011, vol. 52, iss. 49, pp. 6649-6651.

12. Marco Radi, Gian Paolo Vallerini, Alessia Petrelli, Paolo Vincetti, Gabriele Costantino. A one-pot two-step microwaveassisted synthesis of N1-substituted 5,6- ring-fused 2-pyridones. Tetrahedron Letters. 2013, vol. 54, iss.50, pp. 69056908.

13. Weitao Yan, Ruo Wang, Tesen Zhang, Hongtao Deng, Jian Chen, Wei Wua and Zhiqiang Weng. Synthesis of 4trifluoromethyl 2-pyrones and pyridones through the Brønsted base-catalyzed Pechmann-type reaction with cyclic 1,3diones. Org. Biomol. Chem., 2018, vol. 16, pp. 9440-9445.

14. Rasha A. Azzam, Galal H. Elgemeie. Synthesis and antimicrobial evaluation of novel N-substituted 4-ethylsulfanyl-2pyridones and triazolopyridines. Medicinal Chemistry Research. 2019, vol. 28, iss.1, pp. 62-70.

15. Erol D.D., Yulug N. Synthesis and antimicrobial investigation of thiazolinoalkyl-4(1H)-pyridones. European Journal of Medicinal Chemistry. 1994, vol. 29, iss.11, pp. 893-897.

16. Hassan M. Faidallah, Sherif A.F. Rostom, Khalid A. Khan \& Salem A. Basaif. Synthesis and characterization of some hydroxypyridone derivatives and their evaluation as antimicrobial agents. Journal of Enzyme Inhibition and Medicinal Chemistry. 2013, vol. 28, iss.5, pp. 926935.

17. Hayreddin Gezegen, Mustafa Ceylan, Isa Karaman \& Ertan Şahin. Synthesis, Characterization, and Antibacterial Activity of Novel Pyridones. Synthetic Communications. 2014, vol. 44, iss.8, pp. 1084-1093.

\title{
BOZI FUNKSIONALLAȘMIȘ PIRIDON TÖROMOLORININ SINTEZI
}

\author{
F.N. Nă̆lyev \\ Bakı Dövlat Universiteti \\ AZ 1148 Bakl, Z.Xalilov küç., 23; e-mail: farid.orgchemist@gmail.com
}

Metanol mühitindo, metilpiperazinin (MP) iştirakında vo otaq temperaturunda 2-siano-3-(4-piridil)akrilamid ilo benzoilasetonun vo ya asetosirka turşusunun etil efirinin Mixael birlaşmə reaksiyasından müvafiq yeni avazlanmiş piridon töramalarinin amala galdiyi müəyyan edilmişdir. 5-Siano-2-hidroksi-2-metil-6-okso-4-fenilpiperidin-3-karboksil turşusunun etil efirinin 4 saat etil 
spirtindo qaynadilmasindan 5-siano-2-metil-6-okso-4-fenil-1,6-dihidropiridin-3-karboksil turşusunun etil efiri amala galmişdir. Eyni reaksiya şəraitindo 4-brombenzoilasetonitrilin 2siano-3-(2-flüorfenil)akrilamidə Mixael birloşmə reaksiyasından müvafiq piridon törəməsi alınmışdır. Hamçinin, eyni reaksiya şaraitinda 2-siano-3-(4-nitrofenil)akrilamid ila 4-xlorasetoasetat turşusunun etil efirinin qarşılıql tosir reaksiyasindan uyğun piridon törəməsi sintez edilmişdir. Sintez edilmiş bütün birlaşmalarin quruluşları NMR spektroskopiyasının kömayila təsdiq edilmişdir.

Açar sözlər: 2-siano-3-(4-piridil)akrilamid, flüorbenzilidensianoasetamid, 4-xlorasetoasetat efiri, 4brombenzoilasetonitril

\title{
СИНТЕЗ ПРОИЗВОДНЫХ НЕКОТОРЫХ ФУНКЦИОНАЛИЗИРОВАННЫХ ПИРИДОНОВ
}

\section{Ф.H. Нагиев}

Бакинский государственный университет

AZ 1148 Баку, ул. 3.Халилова, 23; e-mail: farid.orgchemist@gmail.com

\begin{abstract}
Выявлено образование новых производных замещенных пиридонов в условиях реакции присоединения по Михаэлю при участии 2-цииано-3-(4-пиридил)акрилалида и бензоилащетона или этилового эфира ацетоуксусной кислоты в присутствии метилпиперазина (МР) в среде метанола. При кипячении в этиловом спирте этилового эфира 5-циано-2-гидрокси2-метил-6-оксо-4-фенилпиперидин-3-карбоновой кислоты в течение 4 часов образуется этиловый эфир 5-цииано-2-метил-6-оксо-4-фенил-1,6-дигидропирид-3-карбоновой кислотыл. В аналогичных условиях были синтезированы соответствующие продукты замещенных пиридонов по реакции присоединения Михаэля взаимодействием 4бромбензоилацетонитрила с 2-цииано-3-(2-фтторфенил)акриламидом и 2-ичиано-3-(4-нитрофенил)акриламида с этилового эфира 4-хлоращетоуксусной кислоты. Структуры всех синтезированных соединений доказаны методом ЯМР-спектроскопии.
\end{abstract}

Ключевье слова: 2-цииано-3-(4-пиридил)акриламид, фторбензилиденцианоацетамид, 4-хлоращетоуксусный эфир, 4-бромбензоилачетонитрил 